\title{
Expression of Oct-4 in oncogenic miR-155-positive oral squamous carcinoma cells
}

\author{
Jun-Song Jiang, Jia Zhu, Hua Han and Qing Wang* \\ Department of Oraland Maxillofacial Surgery, No. 98, Army Hospital, Huzhou, Zhejiang - 313000, PR China
}

*For correspondence: Email: wqwangqing11@hotmail.com; Tel/Fax: 0086-572-3269999

Received: 23 December 2015

Revised accepted: 10 August 2016

\begin{abstract}
Purpose: To identify effective molecular diagnostic methods for oral squamous cell carcinoma (OSCC) to facilitate treatment of the disease in its initial stages.

Methods: To identify molecular markers, OSCC tissue samples were collected from cancer patients and healthy controls. CD44+ cells were sorted using quantitative real-time polymerase chain reaction (qRT-PCR). Immunohistochemistry and immunostaining experiments were performed to identify markers for OSCC.

Results: The qRT-PCR data confirmed the presence of oncogenic miR-155 in the OSCC samples. The immunohistochemical and immunostaining results confirmed the expression of Oct-4, an important target for the early diagnosis of OSCC, in oncogenic miR-155-positive OSCCs.

Conclusion: Detection of the expression of miR-155 and Oct-4, which are key molecular markers, may be useful in improving the early diagnosis of OSCC.
\end{abstract}

Keywords: CD44, oral squamous cell carcinoma, oncogenic miR-155, Oct-4

Tropical Journal of Pharmaceutical Research is indexed by Science Citation Index (SciSearch), Scopus, International Pharmaceutical Abstract, Chemical Abstracts, Embase, Index Copernicus, EBSCO, African Index Medicus, JournalSeek, Journal Citation Reports/Science Edition, Directory of Open Access Journals (DOAJ), African Journal Online, Bioline International, Open-J-Gate and Pharmacy Abstracts

\section{INTRODUCTION}

Oral squamous cell carcinoma (OSCC) is the sixth most prevalent cancer in the world and the third most common cancer in developing countries [1,2]. Further, OSCC is the sixth most common cancer in males and the tenth most common cancer in females [3]. The prognosis of the disease is poor [4]. Therefore, it is important to identify relevant signaling pathways, including molecular markers, for improved diagnosis of the disease [5]. Recently, it was reported that OSCC is functionally heterogeneous [6,7], making it more complex to identify molecular markers that will lead to improved diagnosis. Novel in-depth analysis is essential to diagnose OSCC in the early stages, which is key to overcoming the disease. One study investigated non-coding
RNAs, which have a vital regulatory role in most cancers. In particular, microRNAs (miRNAs) have many regulatory roles, including a major role in the post-transcriptional regulation of various genes [8]. In addition, it has been reported that miRNAs are important in the regulation of various cancers [9]. The transcription factor Oct-4, a member of the POU domain family, is present in pluripotent embryonic stem and germ cells [10-12].

The present study demonstrates the expression of Oct-4 in oncogenic miRNA (miR-155)-positive cells. Detection of the expression of miR-155 and Oct-4, which are key molecular markers for OSCC, may improve diagnosis and facilitate early identification of the disease. 


\section{EXPERIMENTAL}

\section{Sample collection}

In total, 22 OSCC and 22 normal oral tissue samples were collected from patients and healthy volunteers. The tumor samples were collected mostly from the cheek and tongue using a recognized procedure. Immediately after collection, the tumor specimen samples were snap-frozen using liquid nitrogen and stored at $80{ }^{\circ} \mathrm{C}$ until RNA extraction. All biopsy tumor specimens were confirmed to contain > $90 \%$ tumor cells. International guidelines were followed in the study in accordance with the rules set in the Declaration of Helsinki, Finland [13]. Written informed consent was obtained from the patients and in addition approval was also received from the ethical committee of the institute (Department of Oral and Maxillofacial Surgery, Army Hospital, Huzhou, Zhejiang313000, PR China with Ref.No.OMS/22/2016) prior to the start of the study. The institutional Biological Safety Committee monitored the project throughout the studies.

\section{Flow cytometry}

The samples collected from the OSCC patients and controls were labeled with PE mouse antihuman CD44 antibodies (BD Biosciences Pharmingen, San Diego, CA) and detected using a FACSCalibur flow cytometer (Becton Dickinson Immunocytometry Systems, San Jose, CA). Appropriate irrelevant antibodies (isotypes) were used as controls for the flow cytometry experiments.

\section{RNA extraction}

Total RNA was extracted from the two groups of samples using TRIzol reagent (Invitrogen Corp., Carlsbad, CA) as per the manufacturer's instructions. The OSCC samples were homogenized in TRIzol reagent using $1 \mathrm{~mL}$ per 50 to $100 \mathrm{mg}$ of tissue. The concentration and quality of the RNA were determined using a NanoDrop spectrophotometer (Wilmington, DE), and the RNA integrity was assessed by agarose gel electrophoresis (Bio-Rad, Hercules, CA). The isolated total RNA was stored at $-80{ }^{\circ} \mathrm{C}$ for further analysis.

\section{Quantification of miR-34a by quantitative real- time polymerase chain reaction (qRT-PCR)}

The miR-155 level was analyzed using a TaqMan MicroRNA Assay. Total RNA was isolated from the CD44 ${ }^{+}$sorted cells followed by the recovery of small RNA fractions $1<200$ nucleotides) using a mirVANA PARIS miRNA Isolation Kit (Ambion Inc., Foster City, CA). The integrity of the RNA was checked using a NanoDrop spectrophotometer at an absorbance of $260 \mathrm{~nm}$. qRT-PCR was performed using a threshold cycle $(\mathrm{Ct})$ as the fractional cycle number at which the fluorescence exceeds the fixed threshold of 0.2. Quantitative miR-34a expression was analyzed using dCt (the Ct value normalized to internal 'housekeeping' miRNAs such as miR-24 and miR-103) and ddCt (the difference between the $\mathrm{dCt}$ of the positive population and that of the negative population) values for each of the miRNAs. The percentage of expression was calculated using the formula '2 - ddCt'. Total RNA (10 ng) was used to measure miR-155.

\section{Immunohistochemistry}

OSCC tissue samples were collected from cancer patients and subjected to immunohistochemistry. The samples were formalin-fixed and paraffin-embedded using standard protocols. The tissue sections $(7 \mu \mathrm{m})$ were deparaffinized and hydrated. Antigens were retrieved by Tri-sodium citrate treatment $(\mathrm{pH}$ 6.0). Endogenous peroxides and nonspecific immune staining were blocked by hydrogen peroxide and normal serum, respectively. The sections were then incubated overnight at $4{ }^{\circ} \mathrm{C}$ with a monoclonal anti-CD44 ${ }^{+}$antibody. After incubation with the primary antibody, the tissue sections were washed and incubated with horseradish peroxidase-conjugated secondary antibodies. The washed slides were developed with 3,3'-diaminobenzidine (DAB). The prepared slides were counterstained, mounted with DPX, and observed under a microscope.

\section{Immunostaining}

The $\mathrm{CD}_{4} 4^{+}$sorted OSCC samples were fixed with $4 \%$ paraformaldehyde, permeabilized with Triton X-100, and blocked with $1 \%$ bovine serum albumin in phosphate-buffered saline (PBS) for 1 h. The cells were incubated with anti-Oct-4 antibodies (Sigma-Aldrich, St. Louis, MO) overnight at $4{ }^{\circ} \mathrm{C}$. The plates were washed in $1 \times$ PBS and incubated with secondary fluorochrome-conjugated antibodies for $45 \mathrm{~min}$ in the dark and observed using a Nikon Ti-S fluorescence microscope (Tokyo, Japan).

\section{Statistical analysis}

Statistical analysis, including mean, standard deviation, standard error and ANOVA for qRTPCR data, were performed using Microsoft Office Excel 2007 (Microsoft Corp., Redmond, WA). 


\section{RESULTS}

\section{Histological features of OSCC tissue samples}

Molecular markers for the diagnosis of OSCC in the initial stages of the disease are not well established. Identification of the molecular pathways related to OSCC will aid in the design of novel molecular markers for the early and effective detection of OSCC in the initial stages. The present project was designed with this in mind. OSCC tissue samples were collected from the patients as described in the Experimental section using standard procedures and stored accordingly. The OSCC samples were screened using histological techniques. The samples were carefully observed using histological analysis by a pathologist and specialized scientist. All biopsy tumor specimens were observed and confirmed by histological analysis to contain $>90 \%$ tumor cells. The confirmed 22 OSCC samples were further processed for various analyses.

\section{Flow cytometry results}

To isolate OSCC cells from the surrounding tissues, flow cytometry was performed. Normal and OSCC tissue samples were harvested and placed in Dulbecco's modified Eagle's medium. The procedure for cell isolation was performed as described previously with slight modifications. In short, after digestion with collagenase at $37^{\circ} \mathrm{C}$ for $15 \mathrm{~min}$, tissues were squashed and the cell suspension was passed through a $40 \mu \mathrm{m}$ nylon mesh. The processed cells were analyzed by flow cytometric sorting for $\mathrm{CD}_{4} 4^{+}$cells. The results are shown in Fig. 1. The sorted cells from the normal and OSCC tissue samples are shown in Fig $1 \mathrm{~A}$ and $\mathrm{B}$, respectively.

\section{qRT-PCR data}

To study the expression pattern of miR-155, total RNA was isolated from the CD $44^{+}$sorted cells of

$\mathbf{A}$

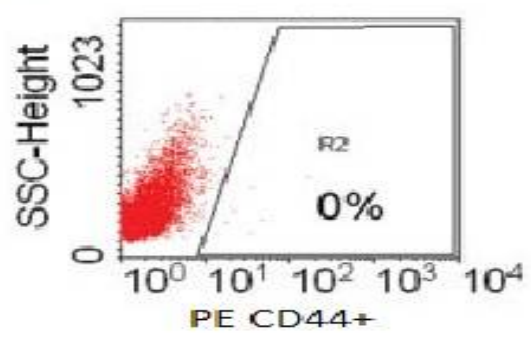

normal and OSCC tissue samples using TRIzol reagent. The integrity of the RNA was checked with a NanoDrop spectrophotometer. For qRTPCR, $10 \mathrm{ng}$ of RNA were used. The qRT-PCR data are shown in Fig 2. The data show the expression profile of miR-155 in normal and OSCC tissue samples. The experiments were repeated thrice with the same samples; the same results were obtained as shown in Fig 2. The relative expression of $\mathrm{miR}-155$ was reduced in normal cells, whereas the level was increased in the OSCC samples. The data are shown in Fig 2.

\section{Immunohistochemistry data}

To identify whether OSCC samples (CD44 ${ }^{+}$cells) express Oct-4, immunohistochemistry was performed. The OSCC tissue and control samples were fixed with $10 \%$ neutral buffered formalin, processed for immunohistochemistry, and stained with anti-Oct-4 and anti-CD44 ${ }^{+}$ antibodies. The data are shown in Fig $3 A$ to $D$. Figure 3B shows $\mathrm{CD}_{4}{ }^{+}$cells in the OSCC samples, whereas Fig. 3D shows no expression of CD44 in the control samples. Figure 3A shows Oct-4-positive cells in the OSCC samples, whereas $\mathrm{Fi}$. 3C shows no Oct-4-positive cells in the control samples.

\section{Immunostaining results}

To confirm and validate that the OSCC samples sorted with $\mathrm{CD}_{4} 4^{+}$cells were Oct-4-positive, immunostaining was performed using anti-Oct-4 antibodies. Oct-4 is a transcription factor and well-known stem cell marker. The $\mathrm{CD}_{4}{ }^{+}$cells were immunostained with anti-Oct-4 antibodies and the plates were examined using a Nikon Ti-S fluorescence microscope; Oct-4-positive cells were observed (Fig 4A). This finding confirms that the OSCC samples sorted with CD44 expressed Oct-4.

B

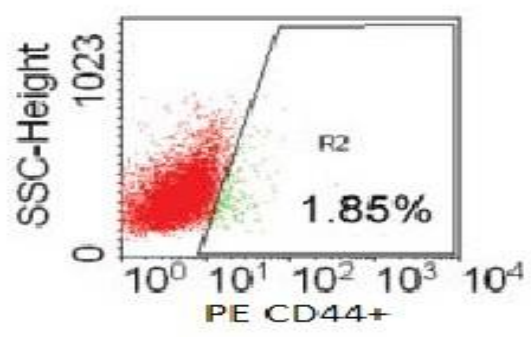

Figure 1: Flow cytometry data. OSCC and normal tissue samples were subjected to cell sorting for CD44 ${ }^{+}$cells. A. In the normal samples, $0 \%$ of the cells were CD $44^{+}$. B. In the OSCC samples, $1.85 \%$ of the cells were CD $44^{+}$ 


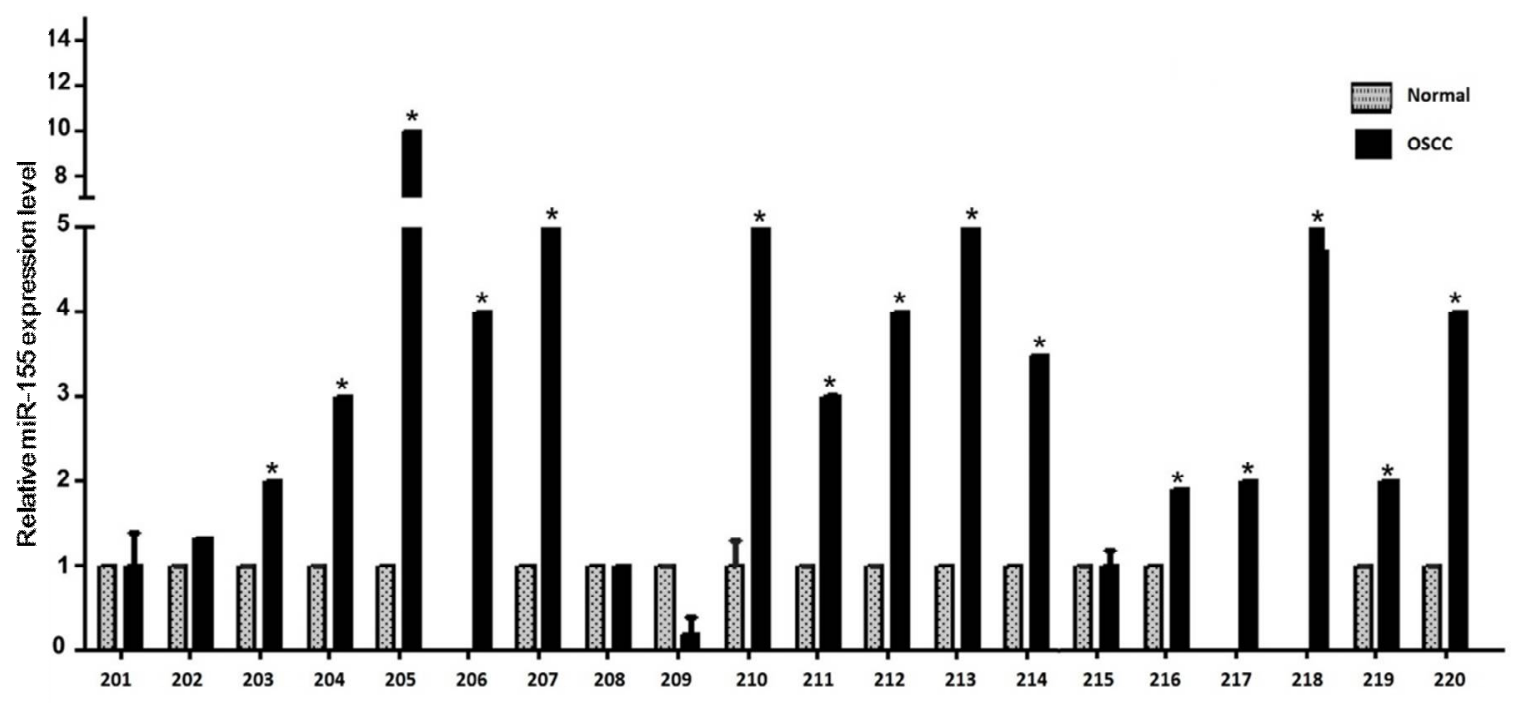

Figure 2: qRT-PCR analysis of the expression of oncogenic miR-155. RNA was isolated from the CD44 ${ }^{+}$sorted cells of OSCC and normal tissue samples and subjected to qRT-PCR. The OSCC cells showed miR-155 expression in all samples when compared with the controls
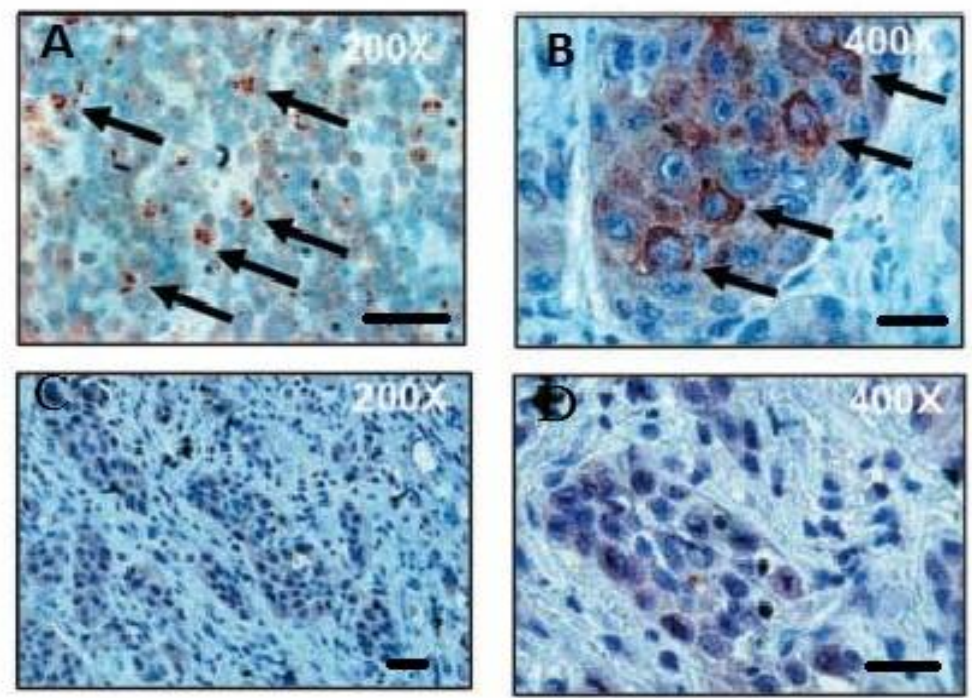

Figure 3: Immunohistochemistry. A. CD44 ${ }^{+}$cells in the OSCC samples appear brown. B. The OSCC samples contained Oct-4-positive cells (indicated by an arrow). C. Normal oral tissue samples showed no CD44 expression. D. Normal oral samples showed no Oct-4 expression. CD44- and Oct-4-positive cells were stained brown using $\mathrm{DAB}$

\section{DISCUSSION}

As stated earlier, OSCC is the sixth most frequent cancer in the world; thus, an effective early diagnostic method is needed to overcome the severity of the disease and provide effective treatment. In the present study, the molecular link between OSCC and Oct-4 was documented and validated. In total, 22 tumor specimen samples were analyzed histologically and confirmed to contain $>90 \%$ tumor cells. The 22 tissue samples used in this study were positive for OSCC.

The present experiment was designed to identify the molecular link between Oct-4 and miR-155 in OSCC. The OSCC positive samples were subjected to flow cytometry to sort $\mathrm{CD} 44^{+}$cells. It has been reported that CD44 and CD133 are cancer stem cell markers [14-16]. The data (Fig. 1) show the flow cytometric analysis of CD44 in both normal and OSCC tissue samples. Interestingly, $\mathrm{CD}_{4} 4^{+}$cells were seen in the 
OSCC tissue samples but not in the control samples. These data illustrate and confirm that the OSCC tissue samples were tumor cells.

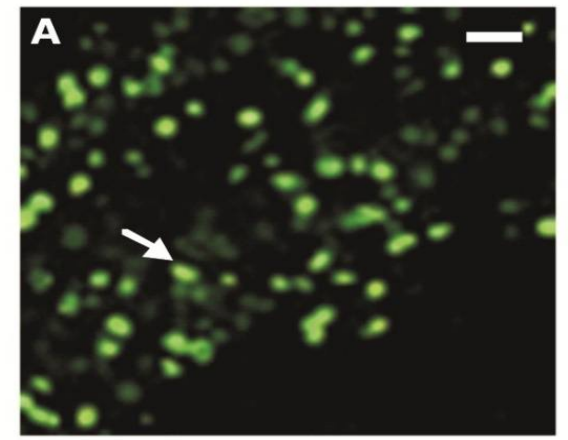

Figure 4: Immunostaining analysis. Immunostaining of the CD $44^{+}$OSCC samples with anti-Oct-4 antibodies produced a fluorescent signal

To identify the expression of oncogenic miR-155 in the $\mathrm{CD}_{4} 4^{+}$sorted OSCC samples, qRT-PCR was performed. The data obtained illustrate that the expression of miR-155 was increased compared with that in the normal samples. The data confirm that oncogenic miR-155 was highly expressed in OSCC patients compared to the controls.

The expression of Oct-4 in CD44 ${ }^{+}$OSCC cells was confirmed by immunohistochemistry. The results clearly show that Oct-4 was expressed in the nucleus. The data also suggest that CD44 is a membrane receptor protein, as the signal was obtained from the border of the cells. Based on the data, it is suggested that OSCC samples express Oct-4. In addition, in accordance with the qRT-PCR and immunohistochemistry data, it was confirmed that oncogenic miR-155 was expressed in the OSCC samples.

To further validate the data, whether the CD44 ${ }^{+}$ cells expressed Oct-4 was determined by immunostaining. CD44 ${ }^{+}$sorted cells from the OSCC samples were used for the immunostaining experiments and stained with anti-Oct-4 antibodies. The results show that the CD $44^{+}$OSCC samples expressed Oct-4. Based on these data, it was confirmed and validated that Oct-4 was expressed in oncogenic miR-155 of OSCC samples. Taken together, the present data reveal a molecular link between miR-155 and Oct-4.

\section{CONCLUSION}

This study paves the way for the development of a new approach for the early diagnosis of OSCC. Detection of the key molecular markers, miR-155 and Oct-4, may be used to improve the diagnosis of OSCC.

\section{DECLARATIONS}

\section{Acknowledgement}

The authors wish to thank the patients who participated in this study for their cooperation.

\section{Conflict of Interest}

No conflict of interest associated with this work.

\section{Contribution of Authors}

The authors declare that this work was done by the authors named in this article and all liabilities pertaining to claims relating to the content of this article will be borne by them.

\section{REFERENCES}

1. Chen YJ, Lin SC, Kao T, Chang CS, Hong PS, Shieh TM, Chang KW. Genomewide profiling of oral squamous cell carcinoma. J Pathol 2004; 204: 326-332.

2. Pentenero M, Gandolfo S, Carrozzo M. Importance of tumor thickness and depth of invasion in nodal involvement and prognosis of oral squamous cell carcinoma: A review of the literature. Head Neck 2005; 27: 1080-1091.

3. Mehrotra R, Yadav S. Oral squamous cell carcinoma: etiology, pathogenesis and prognostic value of genomic alterations. Indian J Cancer 2006; 43: 60.

4. Lo W-L, Kao S-Y, Chi L-Y, Wong Y-K, Chang RC-S. Outcomes of oral squamous cell carcinoma in Taiwan after surgical therapy: factors affecting survival. J Oral Maxillofac Surg 2003; 61: 751-758.

5. Landis SH, Murray T, Bolden S, Wingo PA. Cancer statistics, 1999. CA: A Cancer J Clinicians 1999; 49: 831.

6. Costea D, Tsinkalovsky O, Vintermyr O, Johannessen A, Mackenzie I. Cancer stem cells-new and potentially important targets for the therapy of oral squamous cell carcinoma. Oral Dis 2006; 12: 443-454.

7. Locke M, Heywood M, Fawell S, Mackenzie IC. Retention of intrinsic stem cell hierarchies in carcinoma-derived cell lines. Cancer Res 2005; 65: 8944-8950.

8. Bartel DP. MicroRNAs: genomics, biogenesis, mechanism, and function. Cell 2004; 116: 281-297.

9. Zhang $W$, Dahlberg JE, Tam W. MicroRNAs in tumorigenesis: a primer. Am J Pathol 2007; 171: 728738.

10. Okamoto K, Okazawa H, Okuda A, Sakai M, Muramatsu $M$, Hamada H. A novel octamer binding transcription factor is differentially expressed in mouse embryonic cells. Cell 1990; 60: 461-472. 
11. Rosner MH, Vigano MA, Ozato K, Timmons PM, Poirie F, Rigby PW, Staudt LM. A POU-domain transcription factor in early stem cells and germ cells of the mammalian embryo. Nature 1990; 345: 686-692.

12. Burdon $T$, Smith $A$, Savatier P. Signalling, cell cycle and pluripotency in embryonic stem cells. Trends Cell Biol 2002; 12: 432-438.

13. World MAGA. World Medical Association Declaration of Helsinki: ethical principles for medical research involving human subjects. Journal international de bioéthique= International journal of bioethics 2004; 15: 124.
14. Al-Hajj M, Wicha MS, Benito-Hernandez A, Morrison SJ, Clarke MF. Prospective identification of tumorigenic breast cancer cells. Proc Natl Acad Sci 2003; 100: 3983-3988.

15. Singh SK, Hawkins C, Clarke ID, Squire JA, Bayani J, Hide T, Henkelman RM, Cusimano MD, Dirks PB. Identification of human brain tumour initiating cells. Nature 2004; 432: 396-401.

16. Ricci-Vitiani L, Lombardi DG, Pilozzi E, Biffoni M, Todaro $M$, Peschle C, De Maria R. Identification and expansion of human colon-cancer-initiating cells. Nature 2007; 445: 111-115. 